UDC 622.24.053

DOI: 10.15587/2706-5448.2022.251938

Article type «Reports on Research Projects»

\section{Viktor Svitlytskyi, Irina Boshkova}

\title{
RESEARCH OF THE PULSATING FLOW OF DRILLING FLUID IN THE DRILL STRING
}

The object of research is the pulsating flow of drilling fluid in the drill string. One of the most problematic places is pressure loss due to friction forces distributed along the length of the flow and concentrated in its nodes (threaded joints and pipe bends).

In the course of the study, transformation methods were used that allow the drill string to be represented in the form of straight pipes - elements with distributed parameters connected by different inhomogeneities. This makes it possible to reduce the characteristics of the pulsating flow of the drilling fluid to the determination of the lumped parameters of the inclusions, the limiting conditions at the beginning and end of the drill pipes, as homogeneous sections of the drill string. In turn, pressure losses in the drill string during rotary drilling were divided into two types of losses. These are losses along the entire length of the column (flow) and local pressure losses, which are obtained only in certain places of the liquid flow (for example, tool joints, etc.), due to the fact that the flow suffers local deformation.

It has been found that from a technological point of view, the most favorable well diameter is the one at which the flow resistance in the pipes is equal to the resistance in the annulus. This is due to the fact that during the flow of the drilling fluid, the speed of the turbulent flow decreases only at the walls of the pipe. Therefore, under the action of centrifugal forces on pipe bends, as in heterogeneities, when local pressure losses occur due to separation of the transit flow, the pipe diameter narrows due to the accumulation of solid particles in whirlpool zones and flow velocities. With a smooth turn of the pipe, the specified separation may be absent. In this case, local pressure losses are largely due to the occurrence of a «steam vortex» at the turn (a helical movement caused by the action of inertial forces). Therefore, a necessary condition for rotary drilling is the continuous circulation of the flushing solution, the complete or partial cessation of which makes further drilling impossible. In this case, the drilling process slows down or leads to an accident. This is due to the accumulation of the hard phase in the places where whirlpools appear.

The research results will be useful to scientists and specialists in the oil and gas industry in the physical modeling of well flushing processes in the process of drilling and designing technological flushing processes.

Keywords: drill string, drilling fluid, pulsating flow, hard phase accumulation, rotary drilling.

\section{How to cite}

Svitlytskyi, V., Boshkova, I. (2022). Research of the pulsating flow of drilling fluid in the drill string. Technology Audit and Production Reserves, 1 (1 (63)), 36-40. doi: http://doi.org/10.15587/2706-5448.2022.251938

\section{Introduction}

One of the reasons for the forced vibrations of the drill string elements is the excitation caused by fluctuations in the flow of the drilling fluid [1-3]. With a pulsating flow of drilling fluid, variable loads occur in the drill string. At the same time, they occur when the drilling fluid flows through the interlocks as through local supports. In this case, separation of the transit jet from the pipe walls is formed, as from the main channel, forming the so-called whirlpool areas, interfaces [4-6]. Thus, the force action of the drilling fluid manifests itself in heterogeneities, including string deflections formed in the process of changing the well trajectory (directional drilling) [7-9].

Therefore, it is important to study the processes occurring in the drill string during well flushing.
Thus, the pulsating flow of drilling fluid in the drill string was chosen as the object of research. The aim of research is to study pressure losses due to friction forces distributed along the length of the flow and concentrated in its nodes (threaded connections and pipe bends).

\section{Research methodology}

If to confine ourselves to considering the first five harmonics of the spectrum that generate drilling pumps, then at the minimum sound speed, the wavelength exceeds several meters. Therefore, the linear dimensions of tool joints, which are sufficiently small compared to the wavelength, can be replaced by elements with indirect parameters, for example, baffles, when calculating vibrations in the drill string. Then the drill string can be represented 
in the form of straight pipes - elements with distributed parameters connected by different inhomogeneities. Thus, the determination of the characteristics of the pulsating flow of the drilling fluid is reduced to the determination of the lumped parameters of the inclusions, the limiting conditions at the beginning and end of the drill pipes, as homogeneous sections of the drill string [9-11].

Let's consider the loss of pressure in the drill string during rotary drilling. In this case, two types of losses can be distinguished:

1) losses along the entire length of the column (flow);

2) local pressure losses obtained only in certain places of the fluid flow (for example, lock connections, etc.), due to the fact that the flow in them suffers local deformation.

\section{Research results and discussion}

Let $h_{l}$ denote the pressure loss along the flow length $l$, and let $h_{j}$ denote each local pressure loss.

In Fig. 1 there is a schematic representation of the drill string, consisting of sections of different curvature and elements (drill pipes) connected by threaded locks, which are special units. In addition, there will be pressure losses in the flow that moves through the drill pipes due to friction along the length of the entire string. In the general case, for the drill string, taking into account the pressure losses in its curved sections, it is possible to write:

$$
h_{f}=\sum h_{l}+\sum h_{j}
$$

where $h_{f}$ - total pressure loss along the entire length of the drill string.

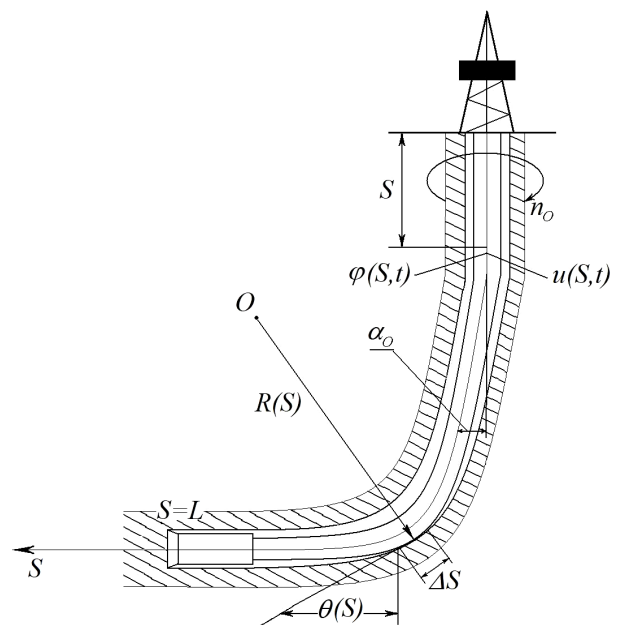

Fig. 1. Schematic representation of the drill string, consisting of sections of different curvature and pipes connected by threaded couplings

In Fig. $1 n_{0}=\partial \varphi / \partial t$ at $S=0 ; n_{0}=$ const; $n_{0} \neq 0-$ rotor rotation; $n_{0}=0$ - downhole motor drilling; $\varphi(S, t)$ - angle of rotation of the current section of the column; $u(S, t)$ - axial displacement of the current section of the column; $L$ - length of the drill string.

It can be said that the value of pressure loss $h_{f}$ is a measure of the mechanical energy of the drilling fluid, which is converted into heat and irretrievably lost by the flow. This is due to the work of friction forces distributed along the length of the flow and concentrated in its nodes (threaded connections and pipe bends).
The greater the friction force in the drilling fluid, the greater, other things being equal, the $h_{f}$ value. There is a certain relationship between the friction forces in a fluid and pressure losses, which is described by the corresponding dependencies [12]. These losses in the first approximation have to be determined using formulas related to the case of established uniform motion with the introduction of correction factors.

Let's determine the dependence of pressure loss along the length of the section between two locks. Let's imagine in Fig. 2 a pipe between two locks, the length of which is $l$.

Here let's direct the flow of the drilling fluid. In the case of uniform fluid motion, the piezometric line $P P$ is an inclined straight line, while its fall along the length of the pipe expresses the pressure loss $h_{l}$.

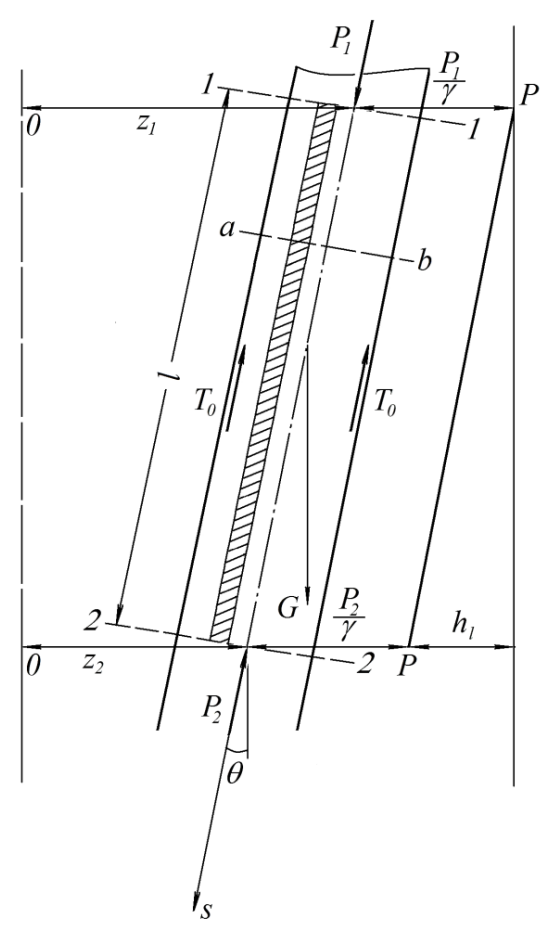

Fig. 2. Forces acting on a part of the flow in an inclined pipe section between two locks

Let's consider the forces acting on the selected part of the fluid flow:

1. Self weight of this part of the wash solution:

$G=\omega l \gamma$

where $\omega$ - open area.

Self-weight projection on the $s$-axis:

$$
G_{s}=\omega l \gamma \cos \theta
$$

where $\theta$ - angle of inclination of the axis with the vertical.

Fig. 2 shows that because:

$$
G_{s}=\omega \gamma\left(z_{1}-z_{2}\right) \text {. }
$$

2. The pressure forces $P_{1}$ and $P_{2}$ are projected onto the $s$ axis in real value and characterize from the side of the rejected parts of the fluid:

$$
P_{1}=p_{1} \omega ; P_{2}=p_{2} \omega,
$$


where $p_{1}$ and $p_{2}$ - the hydraulic pressure at the center of gravity of the living sections $1-1$ and $2-2$.

3 . The projections on the $s$ axis of the normal pressure on the inner surface of the pipe are equal to zero.

4. Friction «force on the wall» $T_{0}$ applied from the side of the pipe walls to the side surface of the flow. This force is directed against the current and is projected onto the axle in real magnitude.

Let's project all the forces on the $s$ axis:

$$
G_{s}+P_{1}-P_{2}-T_{0}=0
$$

Substituting into the resulting equation and the above expressions (4) and (5) and after a series of transformations, we obtain the equation of motion in the given section [12]:

$$
h_{l}=\frac{\tau_{0}}{\gamma} \frac{l}{R}
$$

Apparently, $h_{l}$ for a given drilling fluid and given flow sizes depends only on the average tangential friction stress on the wall $\tau_{0}$.

The force of longitudinal internal friction in a parallelflowing drilling fluid is the force that occurs when individual rectilinear fluid layers slide over each other:

1) directly proportional to the velocity gradient;

2) directly proportional to the surface area of the contacting fluid layers;

3) does not depend on pressure;

4) depends on the physical properties of the drilling fluid, as well as on its temperature (depending on the well depth).

To further determine the energy loss of the turbulent flow (pressure) of the drilling fluid along the length of the drill string, it becomes necessary to describe the design characteristics of its nodes.

The drill string for deep well drilling can be composed of standard drill pipes, drill collars, tool joints, square pipe [13]. DCs (drill collars) have:

a) provide the necessary axial load to obtain a given rate of penetration;

b) stabilize the deflections caused by the total weight.

Drill pipes are seamless seamless pipes with thickened ends due to internal, external or both internal and external upsets. To the ends of the drill pipes are attached on the thread or welded at the joint of the drill locks. The drill joint consists of a coupling and a nipple screwed onto opposite ends of the pipes. The nipple and coupling have locking threads.

From the point of view of hydraulics, the need for a minimum hydraulic resistance, the outer diameter of the locks must correspond to the diameter of the well.

Fig. 3 shows the design of the connection of drill pipes with the help of a lock and a diagram of the flow of flow through this joint.

When a turbulent flow of the drilling fluid flows around the pipe ledge and the end of the thread, a cavity appears (Fig. 3, a), where the flow follows [14, 15]. This leads to separation of the flow from the channel wall. In this case, let's obtain zones filled with whirlpools, where heavy dispersed particles are concentrated (zone $K-$ Fig. $3, b$ ). The deformed flow exiting the top tube enters a larger diameter hole where the flow expands. The deformed (expanded) flow rushes into a pipe with a smaller diameter. In this case, a vortex motion occurs in this zone (zone $K$ - Fig. $3, b$ ).
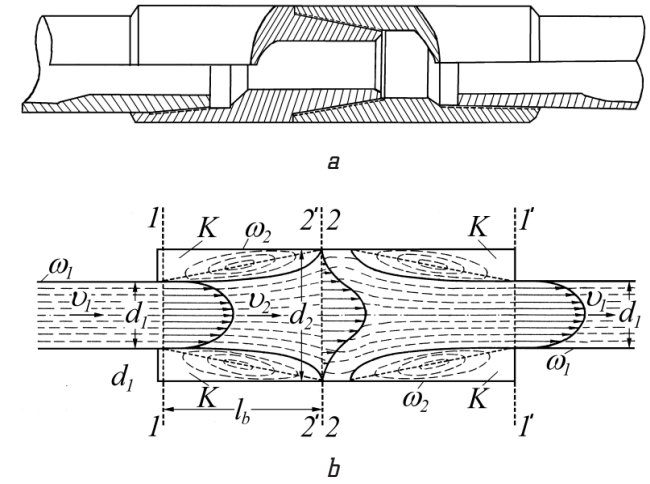

Fig. 3. The design of the connection of drill pipes with the help of a lock and the scheme of flow through this joint: $a$ - lock connection design; $b$ - modeling scheme of the flow of drilling fluid through it

Considering the circuit model (Fig. 3, $b$ ), let's neglect the whirlpool movements in the zones of small extent, due to small losses in them, which will allow to determine the main hydraulic losses in the threaded connection.

Between sections 1-1 and 2-2, local pressure losses $h_{j}$ occur. These pressure losses are due to a sharp expansion of the flow.

To determine local losses, let's use the Borda formula [12]:

$$
h_{j}=\frac{\left(v_{1}-v_{2}\right)^{2}}{2 g} \text {, }
$$

where the difference $\left(v_{1}-v_{2}\right)$ - the speed loss.

According to (8), the loss of pressure corresponds, with a sharp expansion, to the lost speed.

After a series of transformations, formula (8) can be represented as:

$$
h_{j}=\xi_{j}^{\prime} \frac{v_{2}^{2}}{2 g},
$$

where $\xi_{j}^{\prime}$ - drag coefficient for a sharp expansion of the flow.

Formula (9) uses two assumptions:

1) neglect of the forces of external friction in the area between sections $1-1$ and $2-2$, as well as in the area $1^{\prime}-1^{\prime}$ and $2^{\prime}-2^{\prime}$;

2) acceptance of pressure distribution in sections 1-1 and $1^{\prime}-1^{\prime}$ according to the hydrostatic law.

Let's now turn to the local pressure loss that occurs when the flow exits a small diameter pipe (loss «at the outlet»), and denote it by $h_{\text {out }}$.

This case is a special case when $\omega_{2}$ is much less than $\omega_{1}\left(\omega_{2} \ll \omega_{1}\right)$. The pressure loss for the liquid flow at the entrance to the next pipe of the column (significant flow constriction) can be determined by the Borda formula, substituting the drag coefficient $\xi_{\text {out }}$ instead, then:

$$
h_{\text {out }}=\xi_{\text {out }} \frac{v_{2}^{2}}{2 g}
$$

where the coefficient of resistance to a sharp narrowing of the flow at the entrance to the pipe of the flow column $\xi_{\text {out }}$ is equal to $\xi_{\text {out }}=(1 / \varepsilon)-1 ; \varepsilon-$ the flow compression factor.

Next, let's consider local pressure losses for a sharp turn of the drill string when drilling deviated wells.

Fig. 4 shows possible string bends when drilling directional wells. 


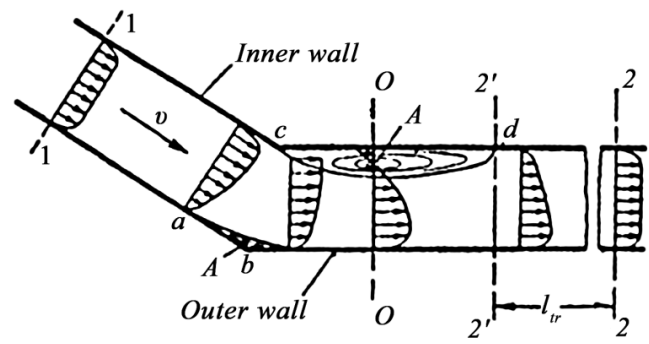

Fig. 4. Calculation scheme of the curvature of the lower part of the drill string during the transition to the horizontal direction of well drilling

This case is characterized by the presence of a $O O$ compressed section of the transit jet and whirlpool sections. As shown above, pressure losses are practically concentrated where there is an expansion of the fluid flow and are written according to the Borda formula in the form:

$$
h_{j}=\xi_{j} \frac{v^{2}}{2 g}
$$

where $\xi_{j}$ - coefficient of local resistance equal to:

$$
\xi_{j}=\left(\frac{\omega}{\omega_{c}}-1\right)^{2}=\left(\frac{1}{\varepsilon}-1\right)^{2},
$$

where $\omega$ - free cross section of the transit jet filling the entire pipe; $\omega_{c}-$ free cross section of the transit jet along the line of the compressed jet of the $O O$ section.

From this formula, it can be seen that the value of $h_{j}$ is directly proportional to the velocity of pressing. The drag coefficient for a given rotation of the bottom of the drill string can be determined according to the data given in Table 1 [12] taking into account the design scheme (Fig. 5).

Table 1

Determination of the loss factor for a sharp turn of the pipe

\begin{tabular}{|c|c|c|c|c|c|c|c|}
\hline$\theta^{\circ}$ & 30 & 40 & 50 & 60 & 70 & 80 & 90 \\
\hline$Z_{\text {s.t. }}$ & 0.20 & 0.30 & 0.40 & 0.55 & 0.70 & 0.90 & 1.10 \\
\hline
\end{tabular}

At the turn of the pipe, let's obtain a curvature of the streamline. Particles of the drilling fluid moving along curved streamlines are subject to centrifugal inertial forces. Due to this force, the hydrodynamic pressure (and, as a result, the potential energy) at the turning point at the outer wall of the pipe increases, and at the inner wall it decreases. This circumstance causes a decrease in high-speed pressing (specific kinetic energy) at the outer wall of the pipe and an increase in it at the inner wall.

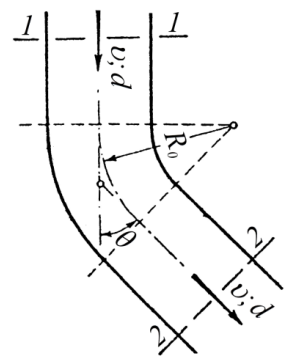

Fig. 5. Calculation scheme of the drill string for directional drilling
The drilling fluid is a two-phase system that absorbs solid particles and bubbles. The bubbles, in turn, are filled with air or vapor of this liquid. This leads to a narrowing of the pipe diameter due to the accumulation of solid particles in the whirlpool zones and to a redistribution of flow velocities. In Fig. 6, an example of the movement of fluid when the pipe is turned at a right angle is shown.

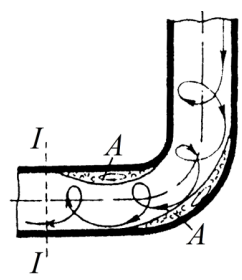

Fig. 6. An example of fluid movement when a pipe is turned at a right angle (transverse circulation)

The separation of the jet from the inner wall increases due to the inertia of the solid particles of the drilling fluid moving near the pipe walls (in the near-wall layer).

The accumulation of the hard phase of the solution in these zones can lead to an accident of the drill string, which was noted during the drilling process.

With a smooth turn of the pipe (directional drilling), the specified separation of the jet may be absent. In this case, local pressure losses are due to the helical movement (vortex) of the drilling fluid.

As it is possible to see, in the central part of the outer wall of the pipe, the pressure will be the highest (due to the high speeds in this part of the pipe).

This position determines the movement of solid particles of the drilling fluid to the right and left (along the outer walls) from the central part to the periphery.

Thus, in order to maintain an efficient rotary drilling process, continuous circulation of the drilling fluid is necessary. Its partial or complete cessation makes the drilling process impossible. In turn, this leads to a slowdown in the drilling process and provokes an emergency situation as a result of the accumulation of the solid phase in the whirlpool zones.

In the future, the study can be used to study the dynamics of the drill string, as a mechanical system with a variable length and with a moving inertial load.

Subsequent studies will consist in the experimental reproduction of the pulsating flow of the drilling fluid, where numerical results will be obtained. The limitation of the studies in the discussed part is the laminar flow regime.

\section{Conclusions}

Summarizing the studies done and analyzing their results, let's confirm that the improvement of drilling technology, an increase in the mechanical speed and depth of wells require more stringent requirements for the movement of the drilling solution from pumps to the bottom.

In the course of the study, it has been found that from a technological point of view, such a well diameter is most favorable, in which the flow resistance in the pipes is equal to the resistance in the annulus.

It has been established that during the flow of the drilling fluid, the turbulent flow velocity decreases only near the pipe walls. Therefore, under the action of centrifugal forces on pipe bends, as in heterogeneities, when 
local pressure losses occur due to separation of the transit flow, the pipe diameter narrows due to the accumulation of solid particles in whirlpool zones and flow velocities.

It has been found that when the pipe is smoothly rotated, this separation may be absent. In this case, local pressure losses are largely due to the occurrence of a «steam vortex» at the turn (helical movement caused by the action of inertial forces).

It has been proven that a necessary condition for rotary drilling is the continuous circulation of the drilling solution, the complete or partial cessation of which makes further drilling impossible. In this case, the drilling process slows down or leads to an accident. This is due to the accumulation of the hard phase in the places where whirlpools appear.

The research results will be useful to scientists and specialists in the oil and gas industry in the physical modeling of well flushing processes in the process of drilling and designing technological flushing processes.

\section{Acknowledgements}

The authors express their gratitude and sincere gratitude to the Doctor of Technical Sciences, Professor Petro Ohorodnikov for his invaluable contribution to the work on the article. And they are deeply despondent about his passing into eternity.

\section{References}

1. Hongtu, Z., Ouya, Z., Botao, L., Jian, Z., Xiangyu, X., Jianping, W. (2021). Effect of drill pipe rotation on gas-solid flow characteristics of negative pressure pneumatic conveying using CFDDEM simulation. Powder Technology, 387, 48-60. doi: http:// doi.org/10.1016/j.powtec.2021.04.017

2. Chudyk, I. I., Dudych, I. F., Tokaruk, V. V. (2020). Well washing process modelling. Prospecting and Development of Oil and Gas Fields, 2 (75), 62-68. doi: http://doi.org/10.31471/19939973-2020-2(75)-62-68

3. Davidenko, A. N., Ignatov, A. A., Polischuk, P. P. (2016). Transportirovka produktov razrusheniia pri burenii skvazhin. Dnepropetrovsk: NGU, 116.
4. Keshavarz Moraveji, M., Sabah, M., Shahryari, A., Ghaffarkhah, A. (2017). Investigation of drill pipe rotation effect on cutting transport with aerated mud using CFD approach. Advanced Powder Technology, 28 (4), 1141-1153. doi: http://doi.org/10.1016/ j.apt.2017.01.020

5. Pang, B., Wang, S., Jiang, X., Lu, H. (2019). Effect of orbital motion of drill pipe on the transport of non-Newtonian fluid-cuttings mixture in horizontal drilling annulus. Journal of Petroleum Science and Engineering, 174, 201-215. doi: http:// doi.org/10.1016/j.petrol.2018.11.009

6. Fylymonenko, N. T., Karakozov, A. A. (2007). Dvyzhenye shlama v pulsyruiushchem vzvesenesushchem potoke, tsyrkulyryruiushchem v pryzaboinoi zone skvazhyni. Naukovi pratsi Donetskoho natsionalnoho tekhnichnoho universytetu. Seriia: Hirnycho-heolohichna, 6, 125-130.

7. Gukasov, N. A., Briukhovetskii, O. S., Chikhotkin, V. F. (1999) Gidrodinamika v razvedochnom burenii. Moscow: OOO «NedraBiznestsentr», 304.

8. Shischenko, R. I., Esman, B. I. (1966). Prakticheskaia gidravlika v burenii. Moscow: Nedra, 285.

9. Iunin, E. K., Khegai, V. K. (2004). Dinamika glibokogo bureniia. Moscow: Nedra, 285.

10. Saroian, A. E. (1979). Burilnye kolonny v glubokom burenii Moscow: Nedra, 229

11. Ogorodnikov, P. I. (1991). Upravlenie uglubleniem skvazhiny na baze izucheniia dinamicheskikh protsessov v burilnoi kolone. Moscow: MINKH i GP im. ak. Gubkina I. M., 463.

12. Chugaev, P. P. (1982). Gidravlika. Leningrad: Energiia, 672.

13. Allikvander, E. A. (1969). Sovremennoe glubokoe burenie. Moscow: Nedra, 228

14. Idelchik, I. E. (1954). Gidravlicheskie soprotivleniia. (Fizikomekhanicheskie osnovy). Moscow: Gosenergoizdat, 316.

15. Zegazhdoi, A. P. (1957). Gidravlicheskie poteri na trenii v kanalakh i truboprovodakh. Moscow: Gosstroiizdat, 278.

Viktor Svitlytskyi, Doctor of Technical Sciences, Professor, Department of Oil and Gas Technologies, Engineering and Heat Power Engineering, Odessa National Technological University, Odessa, Ukraine, ORCID: https://orcid.org/0000-0003-4778-0414

$\triangle$ Irina Boshkova, Doctor of Technical Sciences, Professor, Department of Oil and Gas Technologies, Engineering and Heat Power Engineering, Odessa National Technological University, Odessa, Ukraine, e-mail: boshkova.irina@gmail.com, ORCID: http://orcid.org/ 0000-0001-5989-9223

$\bowtie$ Corresponding author 